\title{
Erythrocyte-Camouflaged Mesoporous Titanium Dioxide Nanoplatform for an Ultrasound-Mediated Sequential Therapies of Breast Cancer
}

\author{
Qunying Li' \\ Bin Lin (iD ${ }^{2}$ \\ Yongzhou $\mathrm{Li}^{3}$ \\ Nan Lu ${ }^{2}$ \\ 'Department of Ultrasound, The Second \\ Affiliated Hospital, Zhejiang University \\ School of Medicine, Hangzhou, Zhejiang, \\ People's Republic of China; ${ }^{2}$ Department \\ of Radiology, The Second Affiliated \\ Hospital, Zhejiang University School of \\ Medicine, Hangzhou, Zhejiang, 310009, \\ People's Republic of China; ${ }^{3}$ Department \\ of Surgery, The Second Affiliated \\ Hospital, Zhejiang University School of \\ Medicine, Hangzhou, Zhejiang, People's \\ Republic of China
}

Correspondence: Nan Lu Department of Radiology, The Second Affiliated Hospital, Zhejiang University School of Medicine, Hangzhou, Zhejiang, 310009, People's Republic of China

Tel/Fax +86/3674665528

Email2317075@zju.edu.cn
Background: The hypoxic microenvironment promotes tumor resistance to most treatments, especially highly oxygen-dependent sonodynamic therapy (SDT).

Method and Results: In view of the aggravation of hypoxia by oxygen consumption during SDT, a biomimetic drug delivery system was tailored to integrate SDT with hypoxiaspecific chemotherapy. In this system, mesoporous titanium dioxide nanoparticles (mTNPs) were developed to deliver the hypoxia-activated prodrug AQ4N with high loading efficiency. Subsequently, a red blood cell (RBC) membrane was coated onto the surface of mTNP@AQ4N. RBC-mTNPs@AQ4N inherited the immune escape ability from RBC membranes, thus efficiently reducing the immunological clearance and improving the work concentration. Upon activation by ultrasound (US), mTNPs as sonosensitizers generate reactive oxide species (ROS), which not only induce apoptosis and necrosis but also disrupt RBC membranes to achieve the US-mediated on-demand release of AQ4N. The released AQ4N was activated by hypoxia to convert into toxic products, which effectively supplemented the inefficiency of SDT in hypoxic tissues. Importantly, SDT-aggravated hypoxia further potentiated this hypoxia-specific chemotherapy of AQ4N.

Conclusion: Based on the sequential strategy, RBC-mTNPs@AQ4N exhibited an excellent synergistic therapeutic effect, thus potentially advancing the development of SDT in cancer treatments.

Keywords: sonodynamic therapy, hypoxia, mesoporous titanium dioxide nanoparticle, biomimetic, sequential therapies

\section{Introduction}

Sonodynamic therapy (SDT) is considered a clinically promising cancer treatment due to its minimal invasiveness, high therapeutic depth and controllable characteristics. ${ }^{1-3}$ Under high-frequency ultrasound (US) activation, sonosensitizers could initiate a sonochemical effect to generate reactive oxide species (ROS), which induces the apoptosis and necrosis of tumor tissues. ${ }^{4-6}$ However, current SDT shows a limited therapeutic outcome, especially in the treatment of large solid tumors. This is because the sonochemical process highly requires the participation of oxygen and involves intensive oxygen consumption. ${ }^{7,8}$ Nevertheless, the interior of the tumor is highly hypoxic, which leads to the inability of SDT to eradicate the tumor. ${ }^{9,10}$ Additionally, the clinically existing sonosensitizers are various types of porphyrin derivatives, such as hematoporphyrin and photofrin, which often exhibit poor bioavailability and ultrasonic stability and easily cause skin phototoxicity. ${ }^{11,12}$ 
In comparison to organic sonosensitizer molecules, mesoporous titanium dioxide nanoparticles (mTNPs) have been widely explored as sonocatalysts because of their low phototoxicity, good biocompatibility and high stability in physiological environments. ${ }^{13-15}$ Furthermore, their mesoporous structure and easily modified surface bestow them with excellent drug loading ability to perform multiple functions. ${ }^{16,17}$

In view of the wide existence of hypoxia in tumor tissues, many methods have been exploited to try to alleviate tumor hypoxia to improve therapeutic efficacy. ${ }^{18-23}$ Unfortunately, these methods are limited due to the complicated tumor microenvironment. Instead of overcoming hypoxia, some opposite strategies that make use of hypoxia have been proposed for more effective cancer treatment. ${ }^{24,25}$ Among them, hypoxiaactivated prodrugs have gained great attention due to their highly selective effect on hypoxic cells. ${ }^{26,27}$ As a typical hypoxic-activated prodrug, 1,4-bis([2-\{dimethylamino-N-oxide\} ethyl]amino)5,8-dihy-

droxy-anthracene-9,10-dione (AQ4N) is relatively safe under normal circumstances but can be converted into 1,4-bis[2-(dimethylaminoethyl)-amino]5,8-dihydroxyanthracene-9,10-dione (AQ4), a potent topoisomerase II inhibitor, by reductase in a hypoxic microenvironment, which can substantially strengthen antitumor activity in hypoxic sites. ${ }^{28,29}$ However, AQ4N has weak cell internalization and a short retention time in vivo due to its high aqueous solubility, greatly decreasing its therapeutic efficiency. ${ }^{30-32}$ The development of mTNPs as drug delivery systems to accommodate AQ4N is a feasible method to overcome low cell endocytosis. More importantly, the integration of SDT and AQ4N, which utilizes the aggravation of tumor hypoxia by SDT to activate AQ4N, promises synergistic cancer treatment. However, the effectiveness of this synergistic strategy that combines mTNPs with AQ4N has not yet been sufficiently demonstrated. In addition, mTNPs still suffer from immunological clearance and biological adhesion, which cause a reduction in the circulating time and working concentration and even the risk of blood clot formation.

A feasible strategy to address these issues is constructing biomimetic nanoparticles by coating active cell membranes. Unlike some artificial materials, which often trigger an immune response from the body's immune defense system, natural cell membrane-coated nanoparticles have a good biocompatibility and low immunogenicity. ${ }^{41}$ Owing to the absolute duplication of functional proteins and lipid composition, biomimetic nanoparticles can inherit the specific features from original cells, thus showing excellent potency in escaping the clearance of the immune system, prolonging blood circulation or promoting specific targeting ability to tumor tissues. ${ }^{33-38}$ As the most abundant cells in the human blood, red blood cells (RBCs) can provide rich materials for nanocarriers functionalization. Furthermore, RBCs lack nucleus and organelles, which is very favorable for their extraction and purification. Thus, RBC membranebased nanocarriers have attracted great attention in the field of drug delivery. ${ }^{39,40}$

In this work, red blood cell (RBC) membrane-cloaked mTNPs with AQ4N loading (RBC-mTNP@AQ4N) were designed for synergistic sonodynamic/chemotherapy (Scheme 1). The RBC-mTNPs@AQ4N inherited the immune escape ability from RBC membranes, thus efficiently distributing in tumor tissues after intravenous administration. Moreover, upon activation by ultrasound, RBC-mTNP@AQ4N generated ROS to inhibit tumor progression and significantly increased tumor hypoxia, which sequentially activated AQ4N. Hypoxia-specific chemotherapy effectively supplemented SDT in hypoxic sites. The in vitro and in vivo results demonstrated the synergistic therapeutic effect of hM-mTNPs@AQ4N with few side effects. Our work provides a promising strategy for efficient and safe sonodynamic/chemotherapy for cancer treatment.

\section{Methods}

\section{Preparation of RBC- Membrane Vesicles}

Whole blood was withdrawn from female Balb/c mice and centrifuged at $1000 \mathrm{r} / \mathrm{min}$ for $3 \mathrm{~min}$ at $4{ }^{\circ} \mathrm{C}$ to remove the buffy coat and plasma. Then, the obtained RBCs were washed with hypotonic PBS solution to induce hemolysis and resuspended in $0.25 \times \mathrm{PBS}$ in ice water. After $30 \mathrm{~min}$, the obtained products were centrifuged at $1000 \mathrm{r} / \mathrm{min}$ for 3 min, and the separated pellet in the light pink layer was collected and washed three times with PBS. To prepare the RBC membrane vesicles, the prepared RBC ghosts were disposed with sonication for $10 \mathrm{~min}$, and then extruded to form membrane vesicles using an Avanti Mini-Extruder (Avanti Polar Lipids) installing with $100 \mathrm{~nm}$ polycarbonate membranes. The prepared RBC membrane vesicles were stored at $-4{ }^{\circ} \mathrm{C}$ for further application. 


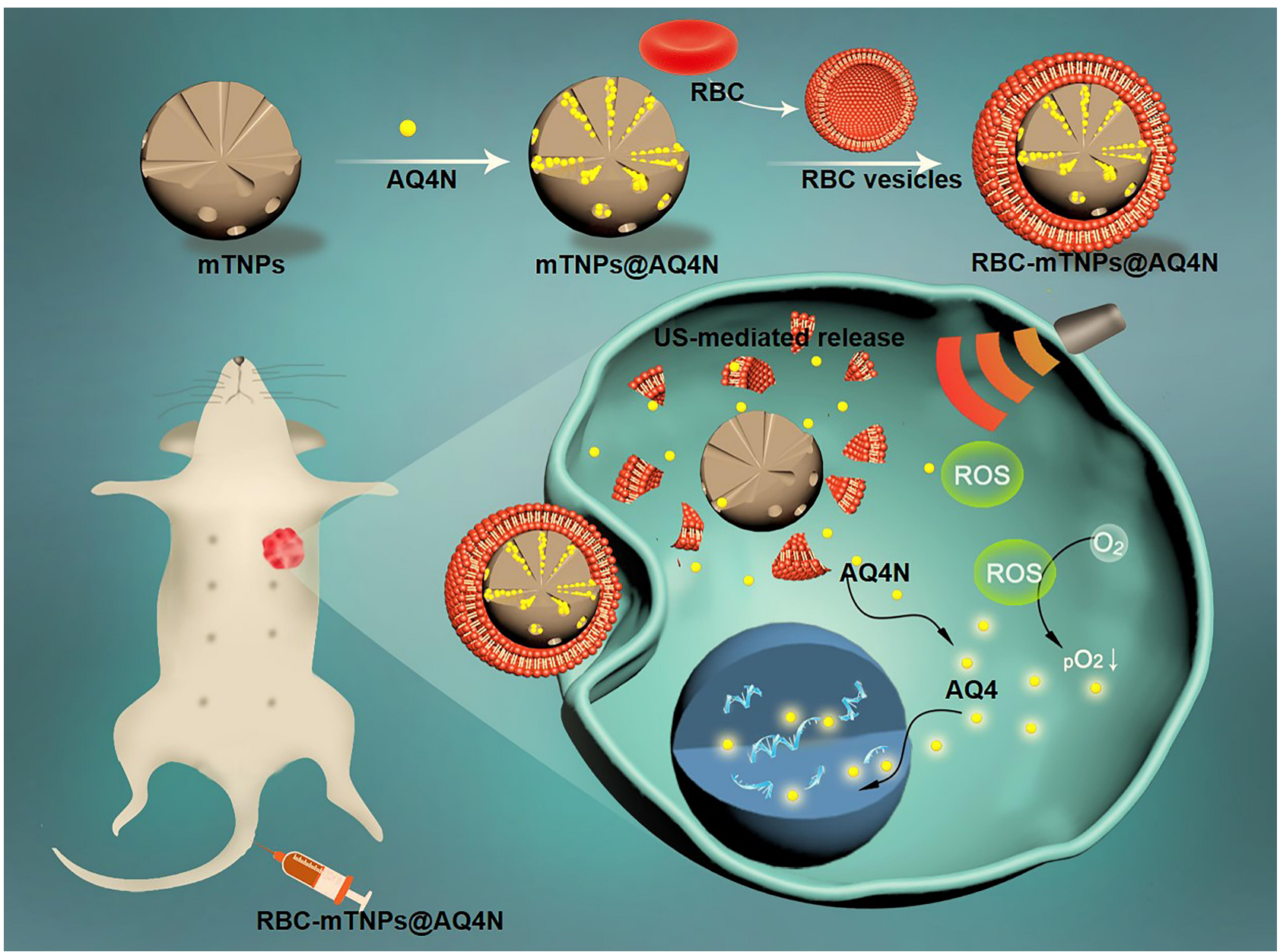

Scheme I A scheme illustration of Red blood cell membrane-coated mesoporous titanium dioxide nanoparticles with hypoxia-activated prodrug loaded, which provided a synergistic strategy for ultrasound-mediated combined sonodynamic/chemotherapy.

\section{Synthesis of mTNPs}

The mTNPs were prepared using a reported method. ${ }^{41,42}$ Briefly, $10 \mathrm{~mL}$ of heptanoic acid was mixed with $50 \mathrm{~mL}$ of ethanol under stirring for $15 \mathrm{~min}$. Then, $0.5 \mathrm{~mL}$ of tetrabutyl titanate was slowly added into the solution under stirring. After $20 \mathrm{~min}, 10 \mathrm{~mL}$ deionized water was added into the mixture and reacted at room temperature for $30 \mathrm{~min}$. Then, the mixture was heated to $120^{\circ} \mathrm{C}$ and reacted for another 1 h. Finally, the products were obtained after centrifugation and repeatedly washed with water and ethanol.

\section{Preparation of RBC-mTNP@AQ4N}

To preload AQ4N into the mTNPs, $5 \mathrm{mg}$ of AQ4N was resuspended in $5 \mathrm{~mL}$ water. Then, $5 \mathrm{mg}$ of mTNPs were added to the aqueous solution of $\mathrm{AQ} 4 \mathrm{~N}$ and mixed under stirring for 24 h. Next, mTNP@AQ4N was obtained after centrifugation at $8000 \mathrm{r} / \mathrm{min}$ for $8 \mathrm{~min}$ and repeatedly washed with water and ethanol. To measure the drug loading content, we collected the remaining AQ4N in the supernatant and quantified it by UV-vis spectroscopy at $610 \mathrm{~nm}$. Then, the drug loading content was determined using the equation: Drug loading content $(\%)=$ mass of AQ4N in mTNP@AQ4N/mass of mTNP@AQ4N. The mass of AQ4N in mTNP@AQ4N was equal to the total mass of $\mathrm{AQ} 4 \mathrm{~N}$ deducted by the mass of $\mathrm{AQ} 4 \mathrm{~N}$ in the supernatant. To coat the RBC membrane onto the nanoparticles,mTNP@AQ4N was mixed with RBC membrane vesicles and sonicated for $20 \mathrm{~min}$. An Avanti MiniExtruder (Avanti Polar Lipids) with $100 \mathrm{~nm}$ polycarbonate membranes was used to extrude the mixture to form RBCmTNP@AQ4N.

\section{Characterization}

The morphology of the nanoparticles was characterized by a Hitachi H-8100IV transmission electron microscope operated at $200 \mathrm{kV}$. Zeta potential and size distribution 
were measured by a Zeta sizer NanoZS (Malvern Instruments, USA). Surface area was detected by the Brunauer-Emmett-Teller (BET) method, and pore diameter distribution was determined by the Barrett-Joyner-Halenda (BJH) method. UV-Vis adsorption was performed by a U-3310 spectrophotometer (Hitachi).

\section{Drug Release}

The release behaviors of RBC-mTNP@AQ4N were investigated using UV-vis spectroscopy and a dialysis method. First, 10 mg of RBC-mTNP@AQ4N was capsulated into dialysis membranes (MWCO 3500) in PBS at $37{ }^{\circ} \mathrm{C}$ with gentle shaking. To verify the ultrasound-controllable release manner, RBC-mTNP@AQ4N was exposed upon external ultrasound $\left(1 \mathrm{~W} \mathrm{~cm}^{-2}, 1 \mathrm{~min}\right)$ at the designed time. Then, released AQ4N in the sample solutions was measured spectrophotometrically at the predetermined time points.

\section{Endocytosis and Intracellular Drug Distribution}

No ethics committee permission was required for the use of MCF-7 cells, MCF 10A and RAW264.7 cells because only certificated cell lines from ATCC were used. Human breast cancer MCF-7 cells and macrophage RAW264.7 cells were cultured in RPMI 1640 medium containing $10 \%$ fetal bovine serum at $37^{\circ} \mathrm{C}$. To evaluate intracellular drug distribution, $2 \times 10^{4} \mathrm{MCF}-7$ cells/well were seeded in 24-well plates and incubated with free AQ4N or RBCmTNP@AQ4N $(25 \mu \mathrm{g} / \mathrm{mL})$ at the same concentration of AQ4N $(5 \mu \mathrm{g} / \mathrm{mL})$ for $6 \mathrm{~h}$. For the RBC-mTNP@AQ4N +US groups, US $\left(1 \mathrm{~W} / \mathrm{cm}^{2} ; 1 \mathrm{MHz} ; 60 \mathrm{~s}\right)$ was applied to stimulate the MCF-7 cells at $2 \mathrm{~h}$ and $4 \mathrm{~h}$ after treatment with RBC-mTNP@AQ4N. Then, these cells were washed three times with PBS and fixed with $4 \%$ paraformaldehyde solution. Then, the nuclei were stained with 4.6-diamino2-phenyl indole (DAPI) for $5 \mathrm{~min}$ and washed with PBS twice. Finally, these cells were observed by laser scanning confocal microscopy, and intracellular AQ4N was quantified by flow cytometry. To investigate the immune-escape ability of RBC-mTNP@AQ4N, RAW264.7 cells were seeded in 24-well plates at a density of $2 \times 10^{4}$ cells/well and incubated with $25 \mu \mathrm{g} / \mathrm{mL}$ FITC-labeled RBC-mTNP @ AQ4N and mTNP@AQ4N for 6 h. Then, the cells were detected using a laser scanning confocal microscope, and the nanoparticles were quantified by flow cytometry.

\section{Cytotoxicity}

MCF-7 cells were planted in 96-well plates $\left(5 \times 10^{3}\right.$ cells/well $)$ in RPMI 1640 cell medium containing $10 \%$ fetal bovine serum at $37{ }^{\circ} \mathrm{C}$. Normoxic MCF-7 cells were cultured under a normoxic condition $\left(74 \% \mathrm{~N}_{2}, 21 \% \mathrm{O}_{2}, 5 \% \mathrm{CO}_{2}\right)$. Hypoxic MCF-7 cells were cultured under hypoxic conditions in a hypoxic incubator with different pressures of $\mathrm{O}_{2}$ and $\mathrm{N}_{2}$ $\left(85 \% \mathrm{~N}_{2}, 10 \% \mathrm{O}_{2}, 5 \% \mathrm{CO}_{2} ; 93 \% \mathrm{~N}_{2}, 2 \% \mathrm{O}_{2}, 5 \% \mathrm{CO}_{2}\right)$. Then, RBC-mTNPs, free AQ4N or RBC-mTNP@AQ4N were administered at the same concentration of free $\mathrm{AQ} 4 \mathrm{~N}$ or RBCmTNPs and coincubated with these cells for $24 \mathrm{~h}$. For the US treatment groups, US $\left(1 \mathrm{~W} / \mathrm{cm}^{2}\right.$; $\left.1 \mathrm{MHz}\right)$ was used to stimulate the MCF-7 cells at $6 \mathrm{~h}$ postadministration. Cell viability was detected using the Cell Counting Kit-8 (CCK-8) assay.

\section{Animal Experiments}

Female nude Balb/c mice were obtained from the Animal Experimental Center of Zhejiang University (6-8 weeks of age). Animal experiments were approved by the Ethics Committee for the Use of Experimental Animals of Zhejiang University. The experimental operation was carried out in agreement with the National Institute of Health Guide for the Care and Use of Laboratory Animals. To establish MCF-7 tumor-bearing nude mice, $1 \mathrm{~mL}$ of MCF-7 cell suspension $\left(2 \times 10^{6}\right)$ was mixed with $1 \mathrm{~mL}$ of Matrigel, and then, the mixture was injected onto the mammary fat pads of female nude Balb/c mice. When the tumor grew to approximately $250 \mathrm{~mm}^{3}, \mathrm{RBC}-\mathrm{mTNPs}(20 \mathrm{mg} / \mathrm{kg})$, free AQ4N (5 mg/kg) and RBC-mTNP@AQ4N (25 mg/kg) were injected into the mice through tail vein. In the US treatment groups, the tumor sites were exposed under US $\left(1 \mathrm{~W} / \mathrm{cm}^{2} ; 1 \mathrm{MHz}\right)$ for $1 \mathrm{~min}$ after $24 \mathrm{~h}$ of intravenous injection and $48 \mathrm{~h}$ of intravenous injection. The tumor volume and body weight were measured every three days. The tumor volume was determined by the equation: Volume $=$ length $\times$ width $^{2} \times 0.52$. On the 23 rd day, the tumors were harvested, imaged and weighed. The major organs, including the heart liver, spleen, kidney and lung, were harvested, fixed and stained with hematoxylin and eosin (H\&E). Blood was collected, and serum biochemistry indexes were measured following the manufacturer's protocol. To detect the hypoxia in tumor tissues, tumor slices were stained with DAPI and antipimonidazole antibodies and observed using confocal microscopy.

To investigate the biodistribution of RBC-mTNP (a)AQ4N, cy5.5-labeled mTNP@AQ4N and RBC-mTNP (a)AQ4N at a dose of $25 \mathrm{mg} / \mathrm{kg}$ were intravenously injected into the MCF-7 bearing female nude mice, 
respectively. The mice were sacrificed at $24 \mathrm{~h}$ postnanoparticle injection and tumor tissues and the main organs including heart, liver, spleen, lung and kidney were harvested, weighted and homogenized. The fluorescence signals were measured to evaluate the biodistribution of the mTNP@AQ4N and RBC-mTNP@AQ4N.

\section{Results and Discussion}

The preparation of RBC-mTNP@AQ4N consists of three steps. (1) The mTNPs were synthesized by a previously reported method. ${ }^{41}$ (2) AQ4N was loaded into the mTNPs by dissolving in aqueous solution and mixing with mTNPs under stirring. (3) RBC membranes were extracted and reconstructed on the surface of RBC-mTNP@AQ4N through a series of extrusions and sonication. As shown in Figure 1A, the prepared mTNPs showed a uniform spherical structure with a diameter of $\sim 40 \mathrm{~nm}$. The analysis of elemental dispersive X-ray (EDX) with elemental mapping indicated the coexistence of titanium and oxygen, and the distribution of these element seems to be homogeneous (Figure 1B). The mesoporous structure could be observed on the surface of mTNPs by high-resolution transmission electron microscopy (Figure S1). Moreover, a typical nitrogen adsorption/desorption isotherm was measured, which further indicated the well-defined mesopores of mTNPs (Figure 1C and Figure S2). The high BET surface area $\left(413.2 \mathrm{~m}^{2} \mathrm{~g}-1\right)$, large pore volume $\left(0.4 \mathrm{~cm}^{3} \mathrm{~g}^{-1}\right)$ and pore size $(2.8 \mathrm{~nm})$ of mTNPs indicated an excellent loading capacity for AQ4N. The loading content of AQ4N was measured by UV-vis spectrophotometry at $610 \mathrm{~nm}$ and was calculated as $20.3 \%$. To improve the stability of mTNPs@AQ4N and prolong its circulating time, $\mathrm{RBC}$ membranes were coated on the surface of mTNP@AQ4N. The TEM images of RBCmTNP@AQ4N in Figure 1D clearly revealed that a grey shell with a thickness of $\sim 10 \mathrm{~nm}$ was around the mTNPs@AQ4N. Dynamic light scattering (DLS) measurements confirmed that RBC-mTNP@AQ4N had a higher size distribution than mTNPs@AQ4N and a surface potential similar to RBC membranes (Figure 1E). Furthermore, sodium dodecyl sulfate-polyacrylamide gel electro-phoresis (SDS-PAGE) analysis indicated that the RBC-mTNP (a)AQ4N contained the specific proteins of RBC membranes (Figure S3). These results confirmed that the RBC membranes were successfully coated onto mTNPs@AQ4N.
A

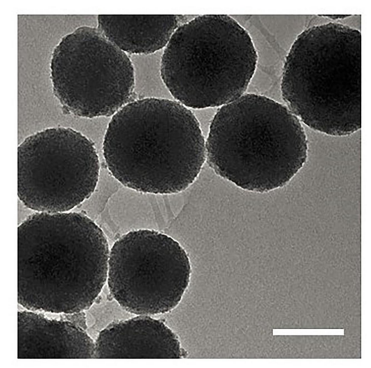

D

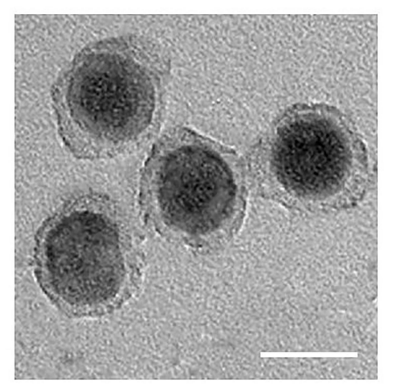

B

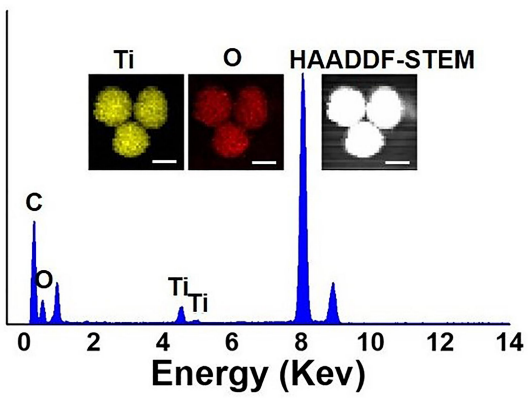

E

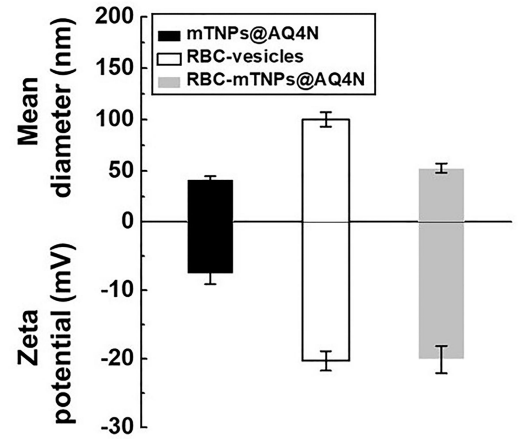

C

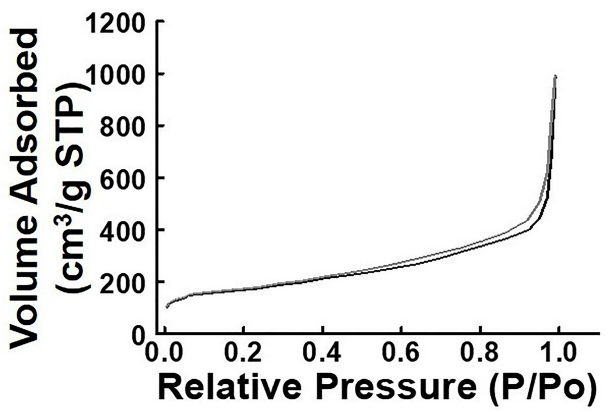

F

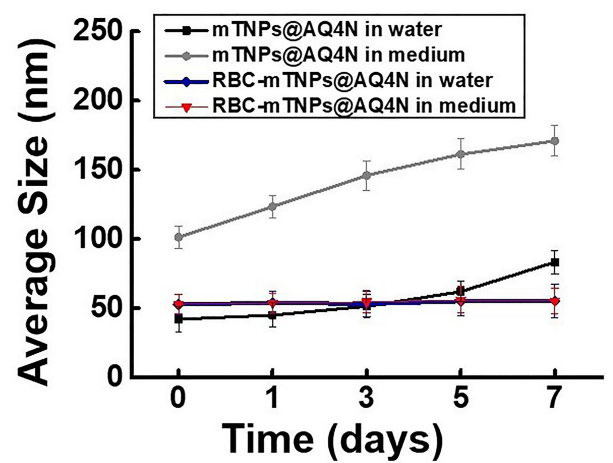

Figure I Characterization of RBC-mTNP@AQ4N. (A) TEM image of mTNPs, scale bar=30nm. (B) Elemental dispersive X-ray (EDX) and elemental mapping of mTNPs, scale bar=30nm. (C) $\mathrm{N}_{2}$ adsorption-desorption isotherms of mTNPs. (D) TEM image of RBC-mTNP@AQ4N, scale bar=50nm. (E) Hydrodynamic diameter and zeta potential of mTNP@AQ4N, RBC vesicles and RBC-mTNP@AQ4N. (F) Average size of various nanoparticles after stocked in PBS and cell medium. The data represent mean \pm S.D. $(n=3)$. 
Additionally, RBC-mTNP@AQ4N showed long-term stability in both water and RPMI-1640 medium with $10 \%$ fetal bovine serum, whereas the aggregation was observed in the cell culture medium containing mTNPs@AQ4N (Figure 1F). The improved colloid stability by the RBC membrane coating probably originated from the feature that homologous RBCs do not form clusters in the blood circulation. ${ }^{43,44}$ To evaluate the ability of RBC-mTNP@AQ4N as a sonosensitizer, we measured the generation of reactive oxide species (ROS) under ultrasound irradiation using singlet oxygen sensor green (SOSG) as an ROS detection reagent. As shown in Figure 2A, neither pure US nor RBCmTNP@AQ4N without US irradiation could produce ROS, whereas RBC-mTNP@AQ4N under US stimulation showed a high amount of ROS generation, which was similar to mTNPs and mTNPs@AQ4N with US. These results indicated that mTNPs and US were crucial for SDT. Based on the generation of ROS, we investigated the integrity of $\mathrm{RBC}$ membranes on the surface of RBC-mTNP@AQ4N. Interestingly, it could be observed that the outside lipid shell of RBC-mTNP@AQ4N was disrupted after irradiation by US, which was probably due to the oxidation and dissociation of the cell membrane triggered by ROS (Figure S4). Since RBC-mTNP@AQ4N could be controllably opened with US activation, it was expected that AQ4N could be released from RBC-mTNP@AQ4N under US stimulation. Therefore, we investigated drug release from RBC-mTNP @AQ4N or mTNPs@AQ4N with or without US exposure. As shown in Figure 2B, the cumulative AQ4N release reached $74.1 \%$ in the mTNPs@AQ4N groups, whereas less than 20\% AQ4N was released from RBC-mTNP@AQ4N without US. Notably, the release of AQ4N from RBC-mTNP @ AQ4N increased to $59.1 \%$ when ultrasound stimulation was carried out at the beginning of the experiment. The USresponsive release property of RBC-mTNP@AQ4N offers the possibility of achieving on-demand cargo delivery under the mediation of US. To further verify the US-controllable drug release of RBC-mTNP@AQ4N, we measured the intracellular distribution of AQ4N in MCF-7 cells using CLSM and FACS analysis. As shown in Figure 2C and D, without US activation, less significant fluorescent signals from AQ4N were detected in MCF-7 cells after incubation with RBC-mTNP@AQ4N than that with mTNP@AQ4N due to the weak release of RBC-mTNP@AQ4N, which led to the quenching of $\mathrm{AQ} 4 \mathrm{~N}$ into the pores as a result of electronic energy transfer. Upon activated by US, RBC-mTNP@AQ4N exhibited remarkably higher intracellular AQ4N fluorescent signals than free $\mathrm{AQ} 4 \mathrm{~N}$, which was approximate to
mTNP@AQ4N. These results confirmed that RBC-mTNP @ AQ4N was an effective drug carrier to enter cells and release AQ4N under US control.

It has been reported that $\mathrm{RBC}$ membrane coatings can endow nanoparticles with immune evasion capability and prolong their circulation lifetime. ${ }^{40,45}$ To evaluate the immune evasion property of RBC-mTNP@AQ4N, RAW264.7 murine macrophages were incubated with RBC-mTNP@AQ4N or mTNP@AQ4N for 6 hrs. As shown in Figure $2 \mathrm{E}$ and $\mathrm{F}$, a large amount of mTNPs@AQ4N was phagocytosed by RAW264.7 cells, leading to high fluorescence signals inside cells. In remarkable contrast, RBC-mTNP@AQ4N showed an extremely weak interaction with RAW264.7 cells due to the RBC membrane markers comprising a number of "don't eat me" signals. The excellent immune evasion capability suggested a prolonged blood circulation time and highly efficient accumulation of RBC-mTNP @ AQ4N in the tumor site.

Encouraged by the outstanding US-activated ROS generation and US-mediated AQ4N release of RBC-mTNP @ AQ4N in cell-free experiments, we sought to investigate the in vitro therapeutic effect. To mimic the tumor microenvironment, MCF-7 cells were cultured under the normoxic condition $\left(21 \% \mathrm{O}_{2}\right)$ and the hypoxic conditions with different degrees $\left(2 \% \mathrm{O}_{2}\right.$ and $\left.10 \% \mathrm{O}_{2}\right)$. The cytotoxicity of various nanoparticles was evaluated using a CCK8 assay kit. As shown in Figure 3A-C, without ultrasound stimulus, RBCmTNPs showed negligible effects on the cell viability of MCF-7 cells under the normoxic condition or the hypoxic conditions, indicating the good biocompatibility of our prepared nanoparticles. In addition, without activation by US, free AQ4N and RBC-mTNP@AQ4N did not induce obvious death of normoxic MCF-7 cells but showed concentrationdependent and hypoxia-dependent cytotoxicity under hypoxic conditions. Notably, RBC-mTNP@AQ4N killed fewer MCF-7 cells than free AQ4N at the same AQ4N concentration and hypoxic conditions, which was probably due to weak drug release of RBC-mTNP@AQ4N without US stimulation. We subsequently investigated the anticancer effect of our nanoparticles upon activation by US. Pure US (1 $\mathrm{W} / \mathrm{cm}^{2} ; 1 \mathrm{MHz} ; 60 \mathrm{~s}$ ) has no obvious effect on cell viability, indicating the biosafety of the applied US (Figure 3D-F). The viability of normoxic MCF-7 cells was remarkably inhibited by US irradiation after treatment with RBCmTNPs and RBC-mTNP@AQ4N. Moreover, RBC-mTNPs showed therapeutic efficiency similar to RBC-mTNP @ AQ4N because of the inefficiency of AQ4N in the 
A
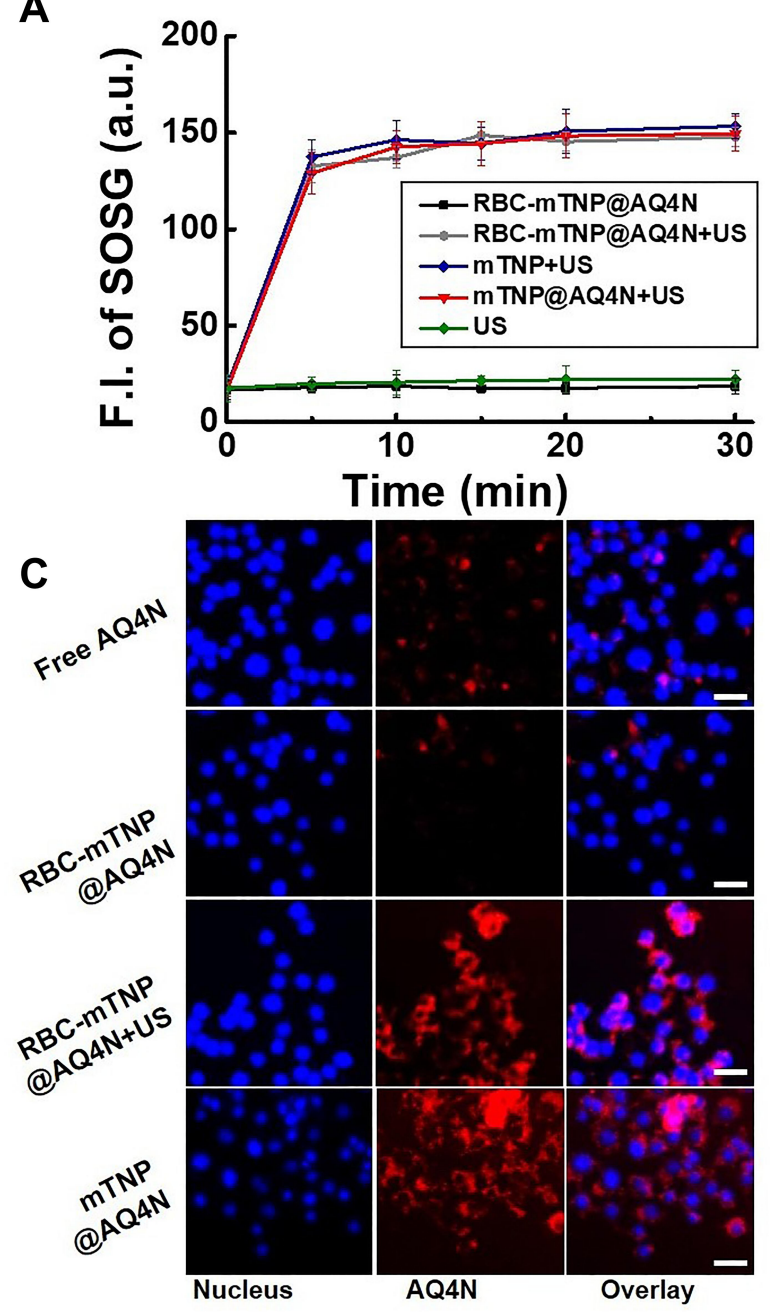

E
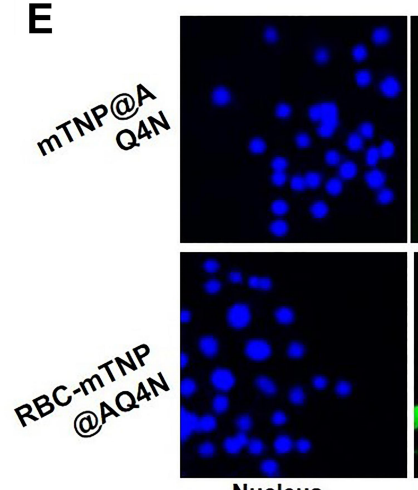

Nucleus
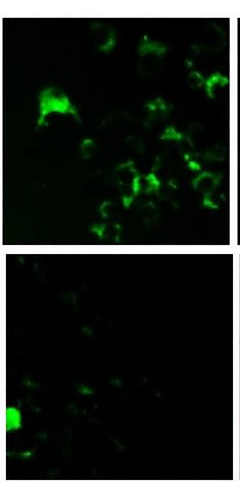

FITC labeled NPs
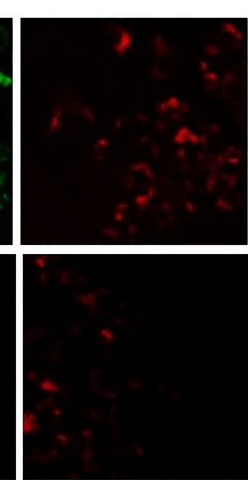

Lysosome
B

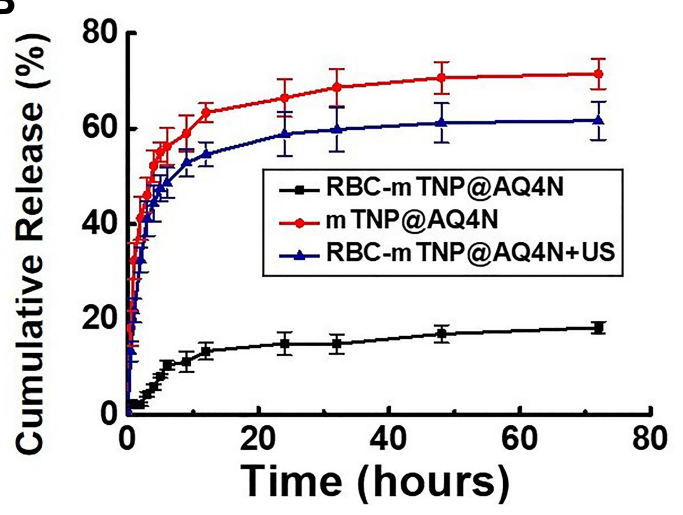

D

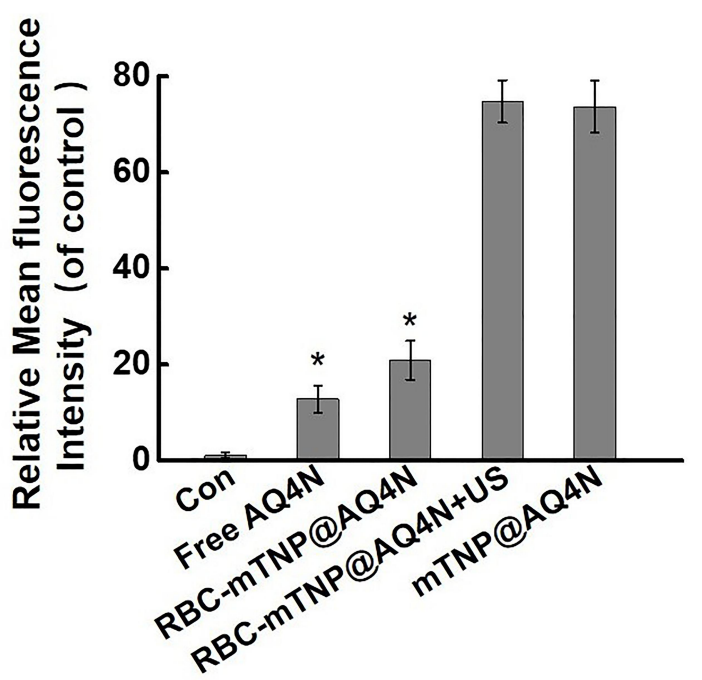

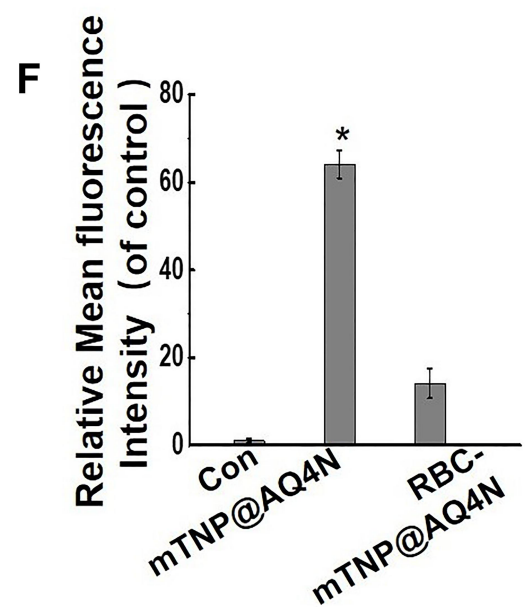

Figure 2 US-mediated drug release behavior. (A) Singlet oxygen generation with or without US irradiation. (B) Cumulative release of AQ4N from mTNP@AQ4N and RBC-mTNP@AQ4N. (C) CLSM observation of intracellular distribution of AQ4N after incubated with free AQ4N and RBC-mTNP@AQ4N for 6 h, scale bars, 10 $\mu$ m. (D) FACS analysis of the relative fluorescence intensity of AQ4N in MCF-7 cells after treated with free AQ4N, RBC-mTNP@AQ4N, or RBC-mTNP@AQ4N plus US for 6 h. The data represent mean \pm S.D. $(n=3)$. *P $<0.05$ vs the RBC-mTNP@AQ4N+US group. (E) Fluorescent microscopic images of RAW264.7 cells after treated with RBCTNP@AQ4N and TNP@AQ4N for $6 \mathrm{~h}$. Green fluorescence signals indicated the location of the FITC-labeled nanoparticles. The lysosomes were stained with Lysotracker Red (red), and the cell nuclei were stained with DAPI (blue). Scale bar= $10 \mu \mathrm{m}$. (F) FACS analysis of the relative fluorescence intensity of FITC-labeled nanoparticles in RAW264.7 cells. The data represent mean \pm S.D. $(n=3)$. *P $<0.05$ vs the RBC-mTNP@AQ4N group. 
A

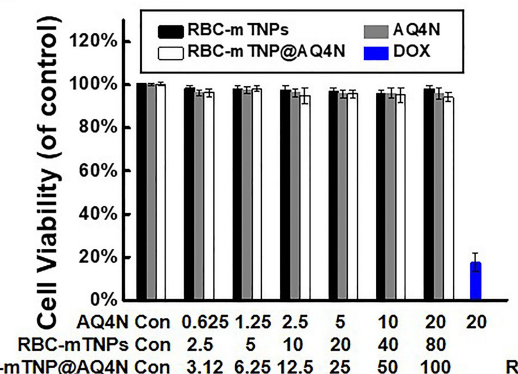

Concentration $(\mu \mathrm{g} / \mathrm{mL})$
B

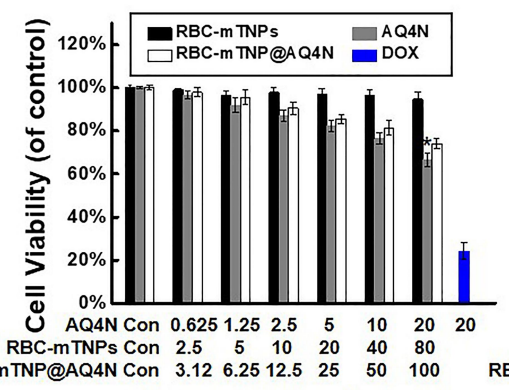

Concentration $(\mu \mathrm{g} / \mathrm{mL})$
C

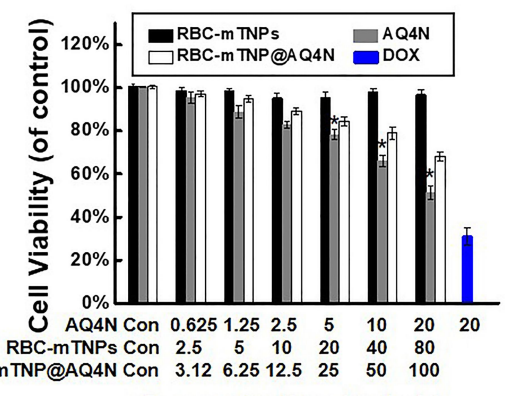

Concentration $(\mu \mathrm{g} / \mathrm{mL})$

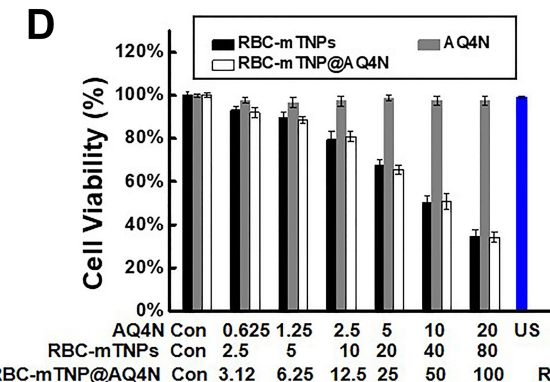

Concentration $(\mu \mathrm{g} / \mathrm{mL})$
E

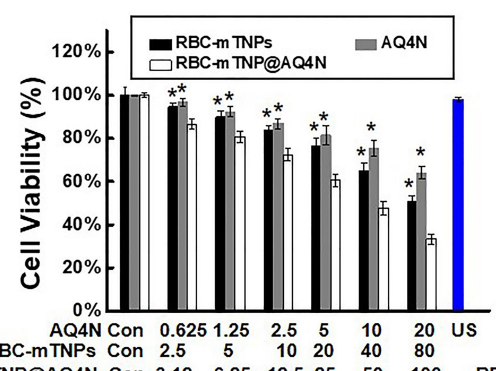

$\begin{array}{lllllll} & \end{array}$

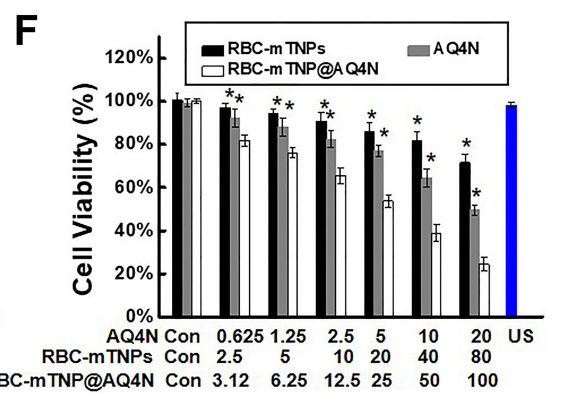

Concentration $(\mu \mathrm{g} / \mathrm{mL})$

Figure 3 In vitro therapeutic effect of RBC-Mtnp@AQ4N. Cytotoxicity of RBC-mTNPs, free AQ4N and RBC-mTNP@AQ4N in MCF-7 cells in 2 I\% pO ${ }_{2}$ condition (A and D), in $21 \% \mathrm{pO}_{2}$ condition (B and $\left.\mathbf{E}\right)$ and in $2 \% \mathrm{pO}_{2}$ condition $(\mathbf{C}$ and $\mathbf{F})$ with $(\mathbf{A}-\mathbf{C})$ or without (D-F) US irradiation. All the data represent mean \pm S.D. ( $\mathrm{n}=6$ ). $* \mathrm{P}<0.05$ vs the RBC-mTNP@AQ4N.

normoxic condition. However, the SDT effect of RBCmTNPs was obviously restricted under hypoxic conditions, and the therapeutic efficiency deceased with decreasing oxygen pressure. It could be interpreted that the sonochemical process exhausted the limited oxygen in the hypoxic microenvironment, which restricted the generation of ROS. Considering the oxygen-dependent features of AQ4N and SDT, we explored the combined sonodynamic/chemotherapy of RBC-mTNP@AQ4N in hypoxic MCF-7 cells. As expected, RBC-mTNP@AQ4N with US showed remarkably higher inhibition effect towards hypoxic MCF-7 cells in comparison to RBC-mTNP@AQ4N without US and RBCmTNPs plus US, confirming the combined effect of the hypoxia-specific chemotherapy and the SDT in this nanoplatform (Figure 3E and F). It was reasonable that RBC-mTNP (a)AQ4N with US generated ROS for SDT and aggravated the hypoxic microenvironment, which improved the therapeutic efficiency of $A Q 4 N$ in a sequential manner, thus achieving a synergistic anticancer effect.

The RBCs coating onto nanoparticles has been demonstrated to remarkably alter their in vivo behavior. ${ }^{46,47}$ Therefore, the biodistribution of these Cy5.5-labeled nanoparticles was first detected $24 \mathrm{~h}$ after intravenous injection.
The mTNP@AQ4N and RBC-mTNP@AQ4N predominantly accumulated in the reticuloendothelial system, including livers, spleens, and kidneys, as well as tumor tissues (Figure 4A). Furthermore, less accumulation in the livers, spleens, and kidneys was observed in the RBCmTNPs@AQ4N- treated groups than in the mTNPs@AQ4N-treated groups. Inspiringly, RBC-mTNPs @AQ4N showed a 1.8-fold higher tumor accumulation than mTNPs@AQ4N.It can be explained that RBCmTNPs@AQ4N had a a reduced retention in the reticuloendothelial system, thus increasing the work concentration in the tumor site. The improved tumor accumulation behavior was consistent with other reported RBC membrane-coated nanoparticles. ${ }^{48}$ To further determine the excellent therapeutic effect of RBC-mTNP@AQ4N, we investigated tumor growth inhibition in $\mathrm{MCF}-7$ tumorbearing nude mice. The MCF-7 tumor-bearing nude mice were randomly divided into eight groups, PBS, RBCmTNPs, AQ4N, mTNP@AQ4N, RBC-mTNP@AQ4N, RBC-mTNPs plus US, mTNP@AQ4N plus US and RBCmTNP@AQ4N plus US, and were intravenously administrated with drugs or/and exposed with US. As shown in Figure 4B-D, the tumor growth in the RBC-mTNP groups 
A
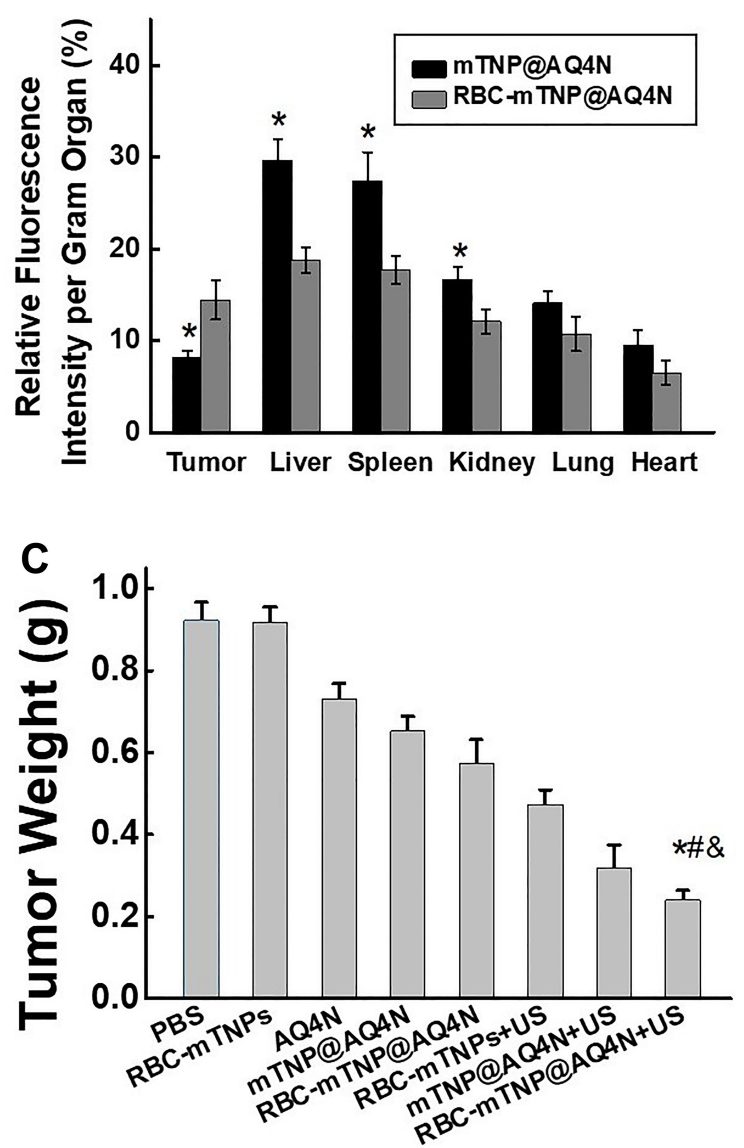

B

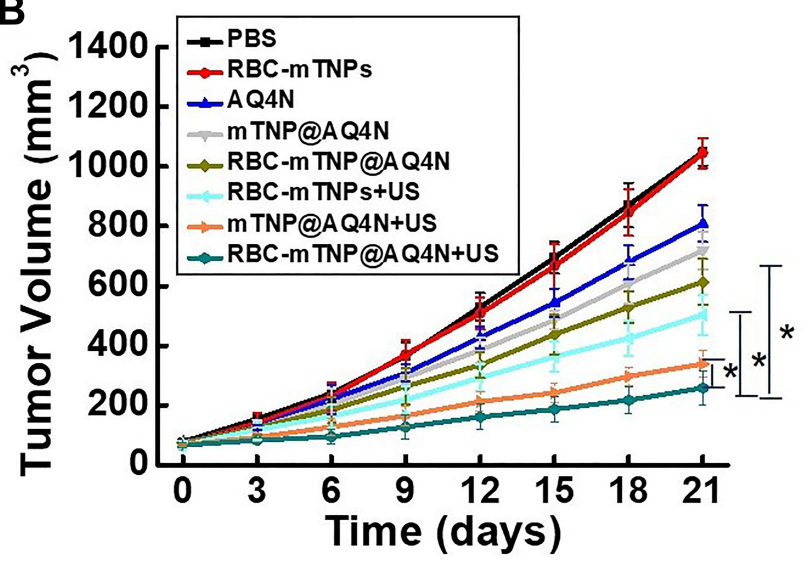

D
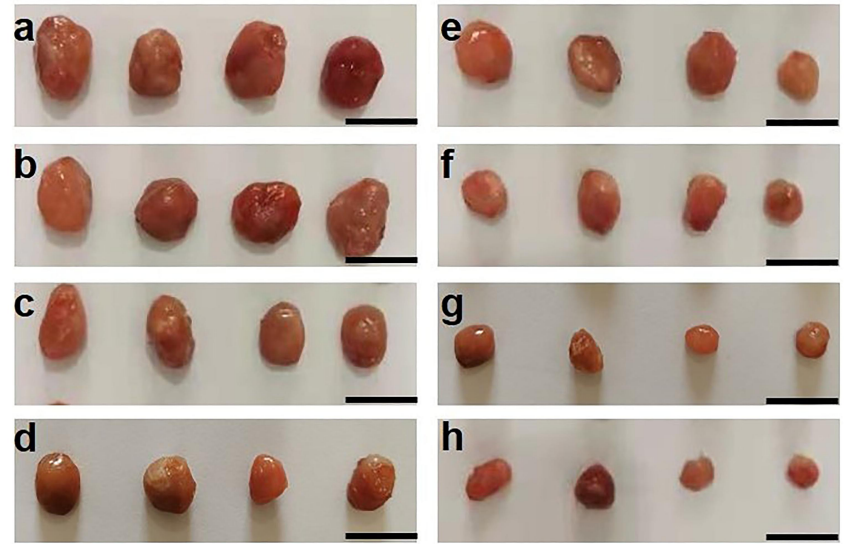

Figure 4 In vivo therapeutic effect. (A) Biodistribution of cy5.5-labeled RBC-mTNP@AQ4N at 24 h of intravenous post-injection. *P < 0.05 vs the RBC-mTNP@AQ4N. (B) Tumor growth curves. (C) Tumor weight. *P $<0.05$ vs the RBC-mTNP + US; ${ }^{\#} \mathrm{P}<0.05$ vs the RBC-mTNP@AQ4N; ${ }^{\text {\& }}<0.05$ vs the mTNP@AQ4N. (D) Tumor photographs.

Notes: a) Control group; b) RBC-TNPs; c) AQ4N; d) mTNP@AQ4N; e) RBC-mTNP@AQ4N; f) RBC-mTNPs+US; g) mTNP@AQ4N+US; h) RBC-TNP@AQ4N+US, scale bar $=I \mathrm{~cm}$. All the data represent mean \pm S.D. $(n=4)$.

was similar to that in the PBS groups, indicating that RBC-mTNPs have little effect on tumor growth. RBCmTNPs@AQ4N showed obviously higher tumor growth inhibition than mTNPs@AQ4N and free AQ4N, which might be attributed to the high tumor accumulation efficiency. In addition, RBC-mTNPs plus US suppressed the tumor volume to a certain extent, whereas RBC-mTNPs @ AQ4N plus US nearly inhibited tumor growth. In particular, RBC-mTNPs@AQ4N plus US exhibited a better therapeutic efficacy than mTNPs@AQ4N plus US, which was consistent with the biodistribution measurement. The excellent therapeutic efficacy of RBC-mTNPs@AQ4N plus US might be contributed to the following mechanism: i) RBC-mTNPs@AQ4N efficiently generated ROS under the US irradiation for the SDT of breast cancer, ii) the released AQ4N from RBC-mTNPs@AQ4N under the ROS stimulus was activated by the hypoxia inside tumors, which supplemented the ineffectiveness of SDT in hypoxic site, iii) the generation of ROS during the SDT aggravated the hypoxia, which further improved the effect of hypoxiaactivated chemotherapy of AQ4N. To validate our hypothesis, we detected the hypoxic status in the tumor tissues after various treatments through immunofluorescence staining. As shown in Figure $5 \mathrm{~A}$ and $\mathrm{B}$, the hypoxiaassociated green fluorescence intensity in the RBCmTNPs@AQ4N-treated groups without US had little change in comparison to that in the control groups, whereas RBC-mTNPs@AQ4N plus US significantly increased the hypoxic signals in the tumor sections, which was approximate to RBC-mTNPs plus US. These results indicated that RBC-mTNPs-based SDT could improve the hypoxia status in tumor tissues. Furthermore, we investigated the apoptotic rates in tumor tissues using TUNEL staining (Figure 5C and D). As we 


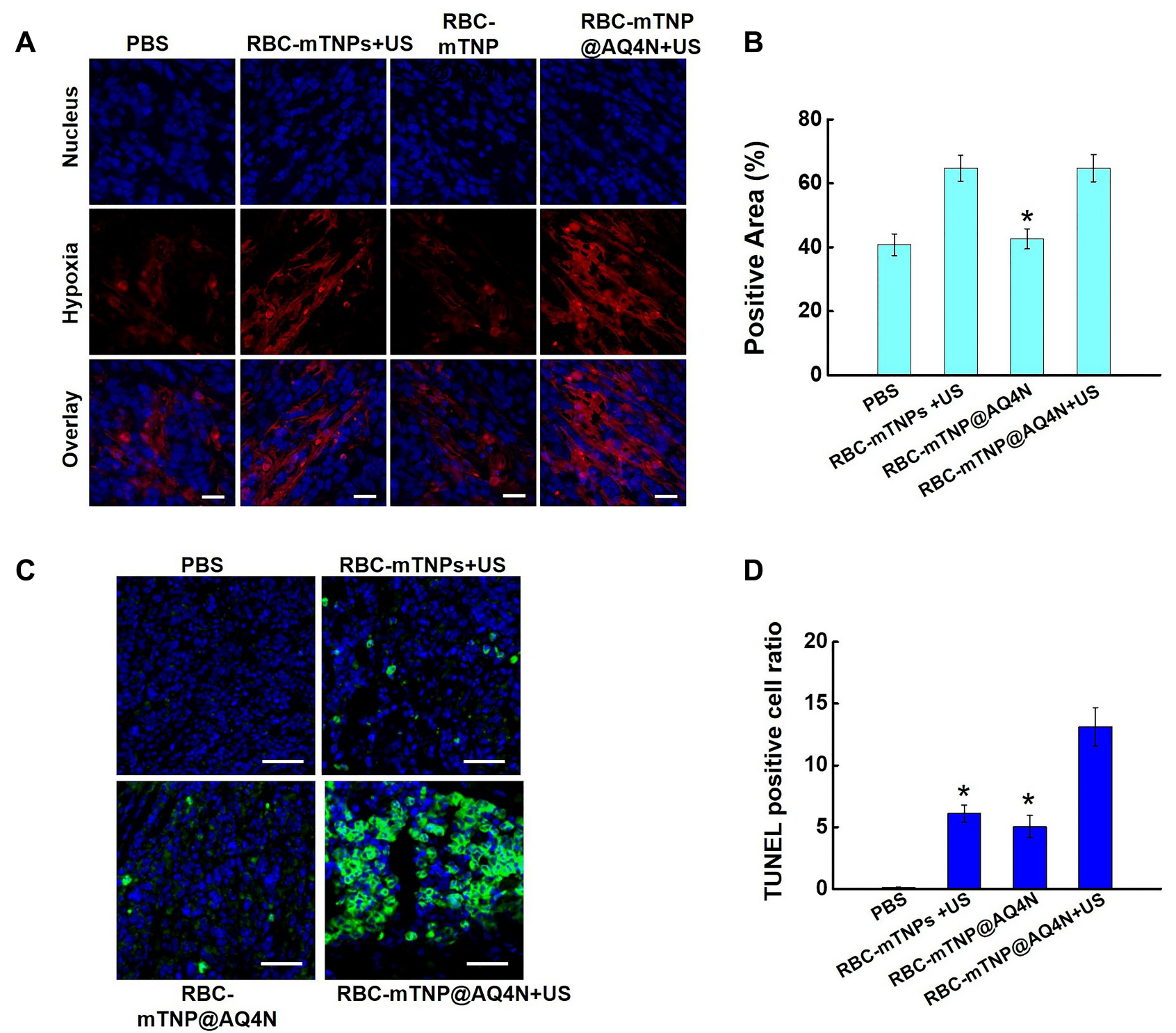

Figure 5 Hypoxic and apoptotic status in tumor tissues. (A) Immunofluorescence images of tumor sections stained with antipimonidazole antibody after various treatments, scale bar $=100 \mu \mathrm{m}$. (B) Flow cytometry analysis of hypoxia positive areas. ${ }^{*} \mathrm{p}<0.05$ versus RBC-mTNP@AQ4N. All the data represent mean \pm S.D. ( $\mathrm{n}=4$ ). (C) Fluorescence images of the TUNEL staining of the tumor sections after various treatments. The blue fluorescence shows the location of the nucleus, and the green fluorescence shows the location of the apoptotic cells; scale bar=100 $\mu \mathrm{m}$. (D) Flow cytometry analysis of tumor apoptotic rate. *P < 0.05 versus RBC-mTNP@AQ4N+US. All the data represent mean \pm S.D. $(n=4)$.

expected, the degree of apoptosis in the RBC-mTNPs @AQ4N plus US groups was remarkably higher than those in RBC-mTNPs@AQ4N groups or RBC-mTNPs plus US groups. Thereby, we believe that RBC-mTNPs @ AQ4N provides a synergistic mechanism for cancer therapy. Compared with other SDT-related nanomaterials, our RBC-mTNPs@AQ4N can release AQ4N under the control of US, and elaborately make use of hypoxia generated by SDT to improve the therapeutic efficiency of AQ4N.
To further explore the potential of hM-mTNPs@AQ4N for clinical application, we carried out a pilot study to evaluate the side effects of the nanoparticles. As shown in Figure S5A, compared with the PBS group, no significant body weight loss was detected in the treatment groups. Furthermore, the serum biochemistry indexes (CRE, ALT, AST, CK and BUN) in all the treatments were maintained at normal levels, and the histological assessment indicated normal physiology in the liver, spleen, kidney, lung, and heart (Figure S5B-F and Figure 6). In this sense, RBC-mTNPs 


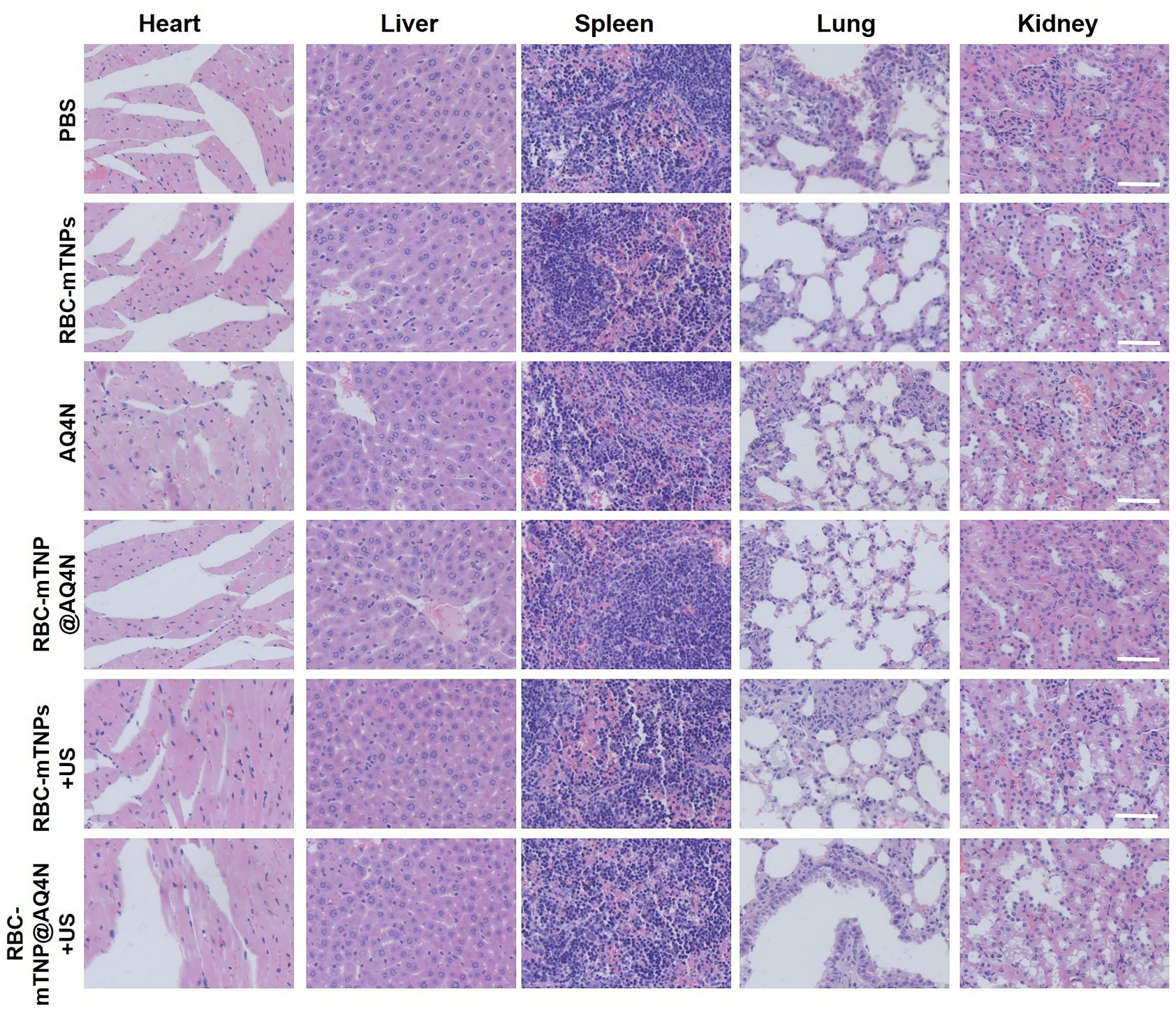

Figure 6 H\&E staining of heart, liver, spleen, lung and kidney from mice after various treatment. Scale bars, $100 \mu \mathrm{m}$.

@ AQ4N would be an effective and safe candidate for chemo/sonodynamic therapy.

\section{Conclusion}

In summary, we developed biomimetic mTNPs with AQ4N loading and RBC membrane coating to combine SDT with hypoxia-specific chemotherapy. Upon activation by US, RBC-mTNP@AQ4N efficiently generated ROS for $\mathrm{SDT}$, further triggering AQ4N release in a controllable manner. The released AQ4N was converted into toxic AQ4 by hypoxia, and SDT-induced hypoxia further improved the anticancer efficiency of AQ4, which supplemented the inefficiency of SDT in hypoxic conditions. In addition, RBC-mTNP@AQ4N showed an immune escape ability due to the coating of the RBC membrane, which effectively reduced retention in the reticuloendothelial system and increased the work concentration in tumor tissues. Thus, RBC-mTNP@AQ4N-based synergistic therapies display an excellent antitumor effect with few side effects. Given their better performance, we believe our designed nanoplatform provides new insight into advancing the development of SDT in the future.

\section{Acknowledgments}

This work was supported by grants from the Zhejiang Science and Technology Project (No. LQ20H180009), the National Natural Science Foundation of China (81901877), the National Science Foundation for Postdoctoral Scientists of China (2018M642460) and the Natural Science Foundation of Zhejiang Province (LQ19H180007). 


\section{Disclosure}

The authors report no conflicts of interest in this work.

\section{References}

1. Trendowski M. The promise of sonodynamic therapy. Cancer Metastas Rev. 2014;33(1):143-160. doi:10.1007/s10555-013-9461-5

2. Costley D, Mc Ewan C, Fowley C, et al. Treating cancer with sonodynamic therapy: a review. Int $J$ Hyperthermia. 2015;31 (2):107-117. doi:10.3109/02656736.2014.992484

3. McHale AP, Callan JF, Nomikou N, Fowley C, Callan B. Sonodynamic therapy: concept, mechanism and application to cancer treatment. In: Therapeutic Ultrasound. Springer; 2016:429-450.

4. Wang X, Zhong X, Bai L, et al. Ultrafine titanium monoxide (TiO1+ $\mathrm{x})$ nanorods for enhanced sonodynamic therapy. $\mathrm{J}$ Am Chem Soc. 2020;142(14):6527-6537. doi:10.1021/jacs.9b10228

5. Gong F, Cheng L, Yang N, et al. Ultrasmall oxygen-deficient bimetallic oxide MnWOX nanoparticles for depletion of endogenous GSH and enhanced sonodynamic cancer therapy. Adv Mater. 2019;31 (23):1900730. doi:10.1002/adma.201900730

6. Liu Y, Wang Y, Zhen W, et al. Defect modified zinc oxide with augmenting sonodynamic reactive oxygen species generation. Biomaterials. 2020;251:120075. doi:10.1016/j.biomaterials.20 20.120075

7. An J, Hu Y-G, Li C, et al. A pH/Ultrasound dual-response biomimetic nanoplatform for nitric oxide gas-sonodynamic combined therapy and repeated ultrasound for relieving hypoxia. Biomaterials. 2020;230:119636. doi:10.1016/j.biomaterials.2019.119636

8. Chen J, Luo H, Liu Y, et al. Oxygen-self-produced nanoplatform for relieving hypoxia and breaking resistance to sonodynamic treatment of pancreatic cancer. Acs Nano. 2017;11(12):12849-12862. doi:10.1021/acsnano.7b08225

9. Zhang N, Tan Y, Yan L, et al. Modulation of tumor hypoxia by $\mathrm{pH}-$ responsive liposomes to inhibit mitochondrial respiration for enhancing sonodynamic therapy. Int J Nanomedicine. 2020;15:5687. doi: $10.2147 /$ IJN.S256038

10. Wang Z, Zhang F, Shao D, et al. Janus nanobullets combine photodynamic therapy and magnetic hyperthermia to potentiate synergetic anti-metastatic immunotherapy. Adv Sci. 2019;6(22):1901690. doi:10.1002/advs.201901690

11. Qian X, Zheng Y, Chen Y. Micro/nanoparticle-augmented sonodynamic therapy (SDT): breaking the depth shallow of photoactivation. Adv Mater. 2016;28(37):8097-8129.

12. Garland MJ, Cassidy CM, Woolfson D, Donnelly RF. Designing photosensitizers for photodynamic therapy: strategies, challenges and promising developments. Future Med Chem. 2009;1 (4):667-691. doi:10.4155/fmc.09.55

13. Harada A, Ono M, Yuba E, Kono K. Titanium dioxide nanoparticle-entrapped polyion complex micelles generate singlet oxygen in the cells by ultrasound irradiation for sonodynamic therapy. Biomater Sci. 2013;1(1):65-73. doi:10.1039/C2BM00066K

14. Ninomiya K, Noda K, Ogino C, Kuroda S-I, Shimizu N. Enhanced $\mathrm{OH}$ radical generation by dual-frequency ultrasound with $\mathrm{TiO} 2$ nanoparticles: its application to targeted sonodynamic therapy. Ultrason Sonochem. 2014;21(1):289-294. doi:10.1016/j.ultsonch.2013.05.005

15. Shi J, Chen Z, Wang B, Wang L, Lu T, Zhang Z. Reactive oxygen species-manipulated drug release from a smart envelope-type mesoporous titanium nanovehicle for tumor sonodynamic-chemotherapy. ACS Appl Mater Interfaces. 2015;7(51):28554-28565. doi:10.1021/ acsami.5b09937

16. Kim S, Im S, Park E-Y, et al. Drug-loaded titanium dioxide nanoparticle coated with tumor targeting polymer as a sonodynamic chemotherapeutic agent for anti-cancer therapy. Nanomedicine. 2020;24:102110. doi:10.1016/j.nano.2019.102110
17. Çeşmeli S, Biray Avci C. Application of titanium dioxide (TiO2) nanoparticles in cancer therapies. J Drug Target. 2019;27 (7):762-766. doi:10.1080/1061186X.2018.1527338

18. Zhu P, Chen Y, Shi J. Nanoenzyme-augmented cancer sonodynamic therapy by catalytic tumor oxygenation. ACS Nano. 2018;12 (4):3780-3795. doi:10.1021/acsnano.8b00999

19. Song M, Liu T, Shi C, Zhang X, Chen X. Bioconjugated manganese dioxide nanoparticles enhance chemotherapy response by priming tumor-associated macrophages toward M1-like phenotype and attenuating tumor hypoxia. ACS Nano. 2016;10(1):633-647. doi:10.1021/ acsnano. 5 b06779

20. Li X, Kwon N, Guo T, Liu Z, Yoon J. Innovative strategies for hypoxic-tumor photodynamic therapy. Angewandte Chemie Int Edn. 2018;57(36):11522-11531. doi:10.1002/anie.201805138

21. Li J, Luo Y, Pu K. Electromagnetic nanomedicines for combinational cancer immunotherapy. Angewandte Chemie Int Edn. 2020.

22. Jiang Y, Li J, Zeng Z, Xie C, Lyu Y, Pu K. Organic photodynamic nanoinhibitor for synergistic cancer therapy. Angewandte Chemie Int Edn. 2019;58(24):8161-8165. doi:10.1002/anie.201903968

23. He S, Jiang Y, Li J, Pu K. Semiconducting polycomplex nanoparticles for photothermal ferrotherapy of cancer. Angewandte Chemie. 2020;132(26):10720-10725. doi:10.1002/ange.202003004

24. Lin W-H, Yeh S-H, Yeh K-H, et al. Hypoxia-activated cytotoxic agent tirapazamine enhances hepatic artery ligation-induced killing of liver tumor in HBx transgenic mice. Proc Natl Acad Sci. 2016;113 (42):11937-11942. doi:10.1073/pnas.1613466113

25. Feng Q, Li Y, Yang X, et al. Hypoxia-specific therapeutic agents delivery nanotheranostics: a sequential strategy for ultrasound mediated on-demand tritherapies and imaging of cancer. $J$ Control Release. 2018;275:192-200. doi:10.1016/j. jconrel.2018.02.011

26. Feng L, Cheng L, Dong Z, et al. Theranostic liposomes with hypoxia-activated prodrug to effectively destruct hypoxic tumors post-photodynamic therapy. ACS Nano. 2017;11(1):927-937. doi:10.1021/acsnano.6b07525

27. Wang Z, Chang Z-M, Shao D, et al. Janus gold triangle-mesoporous silica nanoplatforms for hypoxia-activated radio-chemo-photothermal therapy of liver cancer. ACS Appl Mater Interfaces. 2019;11 (38):34755-34765. doi:10.1021/acsami.9b12879

28. Trédan O, Garbens AB, Lalani AS, Tannock IF. The hypoxia-activated ProDrug AQ4N penetrates deeply in tumor tissues and complements the limited distribution of mitoxantrone. Cancer Res. 2009;69(3):940-947. doi:10.1158/0008-5472.CAN08-0676

29. Albertella MR, Loadman PM, Jones PH, et al. Hypoxia-selective targeting by the bioreductive prodrug $\mathrm{AQ} 4 \mathrm{~N}$ in patients with solid tumors: results of a Phase I study. Clin Cancer Res. 2008;14 (4):1096-1104. doi:10.1158/1078-0432.CCR-07-4020

30. Lalani AS, Alters SE, Wong A, Albertella MR, Cleland JL, Henner WD. Selective tumor targeting by the hypoxia-activated prodrug $\mathrm{AQ} 4 \mathrm{~N}$ blocks tumor growth and metastasis in preclinical models of pancreatic cancer. Clin Cancer Res. 2007;13 (7):2216-2225. doi:10.1158/1078-0432.CCR-06-2427

31. Zhang R, Feng L, Dong Z, et al. Glucose \& oxygen exhausting liposomes for combined cancer starvation and hypoxia-activated therapy. Biomaterials. 2018;162:123-131. doi:10.1016/j. biomaterials.2018.02.004

32. Li C, Yang X-Q, An J, et al. Red blood cell membrane-enveloped O2 self-supplementing biomimetic nanoparticles for tumor imaging-guided enhanced sonodynamic therapy. Theranostics. 2020;10(2):867. doi:10.7150/thno.37930

33. Yue J, Wang Z, Shao D, et al. Cancer cell membrane-modified biodegradable mesoporous silica nanocarriers for berberine therapy of liver cancer. RSC Adv. 2018;8(70):40288-40297. doi:10.1039/ C8RA07574C 
34. Zhu J-Y, Zheng D-W, Zhang M-K, et al. Preferential cancer cell self-recognition and tumor self-targeting by coating nanoparticles with homotypic cancer cell membranes. Nano Lett. 2016;16 (9):5895-5901. doi:10.1021/acs.nanolett.6b02786

35. Liu B, Wang W, Fan J, et al. RBC membrane camouflaged prussian blue nanoparticles for gamabutolin loading and combined chemo/ photothermal therapy of breast cancer. Biomaterials. 2019;217:119301. doi:10.1016/j.biomaterials.2019.119301

36. Zhen X, Cheng P, Pu K. Recent advances in cell membrane-camouflaged nanoparticles for cancer phototherapy. Small. 2019;15 (1):1804105. doi:10.1002/smll.201804105

37. Li J, Zhen X, Lyu Y, Jiang Y, Huang J, Pu K. Cell membrane coated semiconducting polymer nanoparticles for enhanced multimodal cancer phototheranostics. Acs Nano. 2018;12(8):8520-8530. doi:10.1021/acsnano.8b04066

38. Zeng Z, Pu K. Improving cancer immunotherapy by cell membranecamouflaged nanoparticles. Adv Funct Mater. 2020;30(43):2004397. doi:10.1002/adfm.202004397

39. Pierigè F, Serafini S, Rossi L, Magnani M. Cell-based drug delivery. Adv Drug Deliv Rev. 2008;60(2):286-295. doi:10.1016/j. addr.2007.08.029

40. Xia Q, Zhang Y, Li Z, Hou X, Feng N. Red blood cell membrane-camouflaged nanoparticles: a novel drug delivery system for antitumor application. Acta Pharmaceutica Sinica B. 2019;9 (4):675-689. doi:10.1016/j.apsb.2019.01.011

41. Liu S, Han G, Shu M, Han L, Che S. Monodispersed inorganic/ organic hybrid spherical colloids: versatile synthesis and their gas-triggered reversibly switchable wettability. J Mater Chem. 2010;20(44):10001-10009. doi:10.1039/c0jm02101f
42. Guo Z, Zheng K, Tan Z, et al. Overcoming drug resistance with functional mesoporous titanium dioxide nanoparticles combining targeting, drug delivery and photodynamic therapy. J Mater Chem B. 2018;6(46):7750-7759. doi:10.1039/C8TB01810C

43. Xuan M, Shao J, Zhao J, Li Q, Dai L, Li J. Magnetic mesoporous silica nanoparticles cloaked by red blood cell membranes: applications in cancer therapy. Angewandte Chemie Int Edn. 2018;57 (21):6049-6053. doi:10.1002/anie.201712996

44. Gao W, Zhang L. Engineering red-blood-cell-membrane-coated nanoparticles for broad biomedical applications. AiChE J. 2015;61 (3):738-746. doi:10.1002/aic.14735

45. Jiang Q, Luo Z, Men Y, et al. Red blood cell membrane-camouflaged melanin nanoparticles for enhanced photothermal therapy. Biomaterials. 2017;143:29-45. doi:10.1016/j. biomaterials.2017.07.027

46. Rao L, Bu LL, Xu JH, et al. Red blood cell membrane as a biomimetic nanocoating for prolonged circulation time and reduced accelerated blood clearance. Small. 2015;11(46):6225-6236. doi:10.1002/smll.201502388

47. Hao X, Li Q, Wang H, et al. Red-blood-cell-mimetic gene delivery systems for long circulation and high transfection efficiency in ECs. J Mater Chem B. 2018;6(37):5975-5985. doi:10.1039/C8TB01789A

48. Wang C, Sun X, Cheng L, et al. Multifunctional theranostic red blood cells for magnetic-field-enhanced in vivo combination therapy of cancer. Adv Mater. 2014;26(28):4794-4802. doi:10.1002/ adma.201400158
International Journal of Nanomedicine

\section{Publish your work in this journal}

The International Journal of Nanomedicine is an international, peerreviewed journal focusing on the application of nanotechnology in diagnostics, therapeutics, and drug delivery systems throughout the biomedical field. This journal is indexed on PubMed Central, MedLine, CAS, SciSearch ${ }^{\circledR}$, Current Contents ${ }^{\circledR} /$ Clinical Medicine,
Journal Citation Reports/Science Edition, EMBase, Scopus and the Elsevier Bibliographic databases. The manuscript management system is completely online and includes a very quick and fair peer-review system, which is all easy to use. Visit http://www.dovepress.com/ testimonials.php to read real quotes from published authors. 